\title{
Insect Bite Related Lip Swelling
}

\section{Ashaya M*, Raghavendra K, Prasanna KR, Roopashri RK, Ujwala SS and Warrier S}

A J Institute of Dental Sciences, India

*Corresponding author: Ashaya M, A J Institute of Dental Sciences, India, Tel: 7899497464; Email: drashaya0309@gmail.com

\section{Case Report}

Volume 6 Issue 4

Received Date: December 02, 2021

Published Date: December 30, 2021

DOI: $10.23880 /$ oajds-16000320

\section{Abstract}

Insect bite is a common event in a tropical and thickly populated country like India. Insect bite's physical effect on skin receives less attention, especially in the rural regions. An uncommon yet interesting aspect that arises out of diagnosing insect bite mucositis is the similarity it may pose with other mucosal lesions. The effect of bite may last long or lead to undesired lesion in course of treatment quite certainly in case of underlying systemic disorder. This is an article which reports a case of insect bite and traumatic ulcer of lower lip with highlights on pathogenesis, clinical features, effect of underlying systemic condition and treatment options.

Keywords: Insect bite; Traumatic ulcer; Lower lip

\section{Introduction}

A 60 year old male patient visited with the chief complaint of swelling and pain in the lower lip since 15 days. $\mathrm{H} / \mathrm{O}$ insect bite 15 days back followed by sudden onset of swelling in entire lower 3rd of the face overnight $\mathrm{H} / \mathrm{O}$ biopsy of the lowerlip 12 days back. Non-healing ulcer at the biopsy site. Patient was diabetic, he had undergone angioplasty 4 days back. He was on following medications, Tab Metab500 mg, Tab Gopril-5 mg (ramipril), Tab Mono SR 30 mg (isosorbid), Tab Metloc sr-50 mg(metoprolol), Tab clopt 75 mg (clopitab). Tab Ecosprin 150 mg, Mixtard 18 units BID Patient had Smoking habit since 15 yrs 4 to 5 times per day.

Extraoral examination revealed diffuse swelling of lower lip, extending throughout the length of the lip with maximum convexity on the left side. Skin over the swelling was erythematous, stretched and shiny. On palpation there was tenderness and no paresthesia was elicited (Figures 1 \& 2).

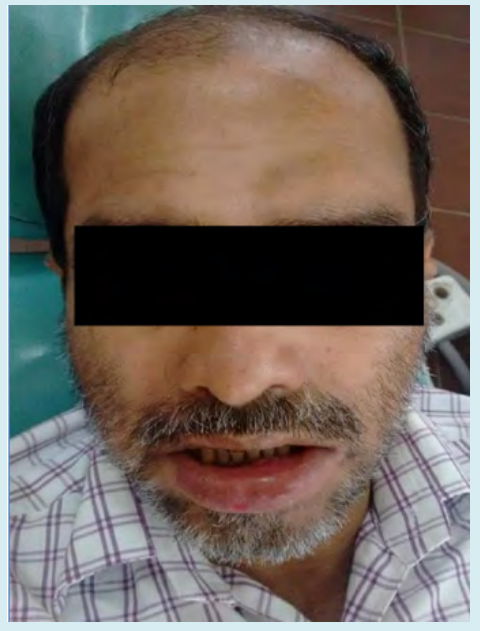

Figure 1: Extraoral photograph. 


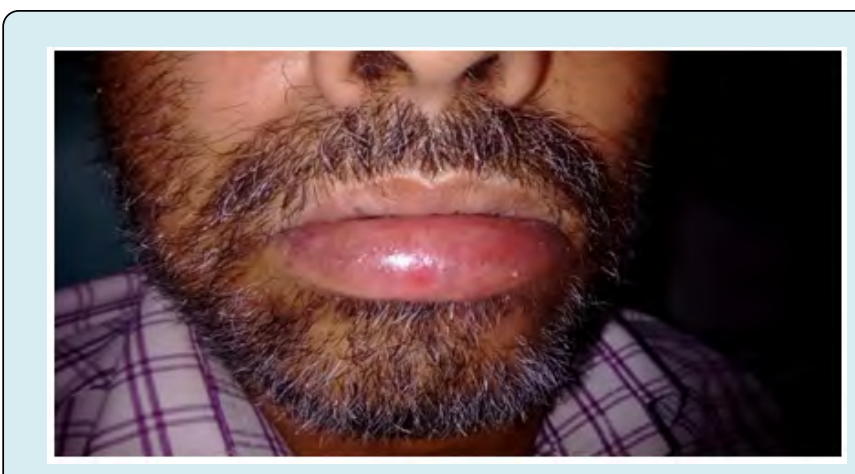

Figure 2: Extraoral image showing diffuse swelling of lower lip.

Intraoral examination revealed diffuse swelling of lip extending to the labial mucosa till the vestibule. There was presence of an ulcer in lower labial mucosa on the left side irregular in shape measuring about $2 \mathrm{~cm} \times 2 \mathrm{~cm}$ in dimension. The margin of the ulcer was well defined with a sloping edge surrounded by erythematous halo. Floor of the ulcer was yellowish white in colour. No pus discharge was seen. Ulcer was tender, swelling was firm in consistency. No paresthesia was elicited (Figure 3).

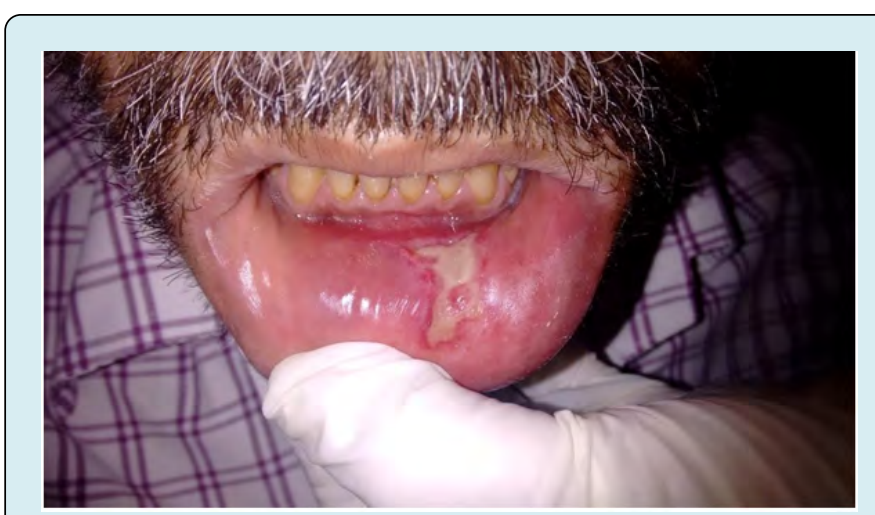

Figure 3: Intraoral image showing well defined nonhealing ulcer in lower lip.

Considering the history and clinical features we arrived at the diagnosis of Insect bite in lower lip and traumatic ulcer in lower labial mucosa Differential diagnosis that can be considered is diabetic ulcer in lower lip. Patient was advised to quit the deleterious habit of smoking as it was seen hindering the process of healing. Tab citirizine was advised once a day. Metrogyl DG forte gel and Zytee gel were prescribed for application over the lesion. Mouth wash with betadine was advised twice daily for 1 week. The prognosis was good. Patient was recalled for follow-up and healing was satisfactory (Figures $4 \& 5$ ).

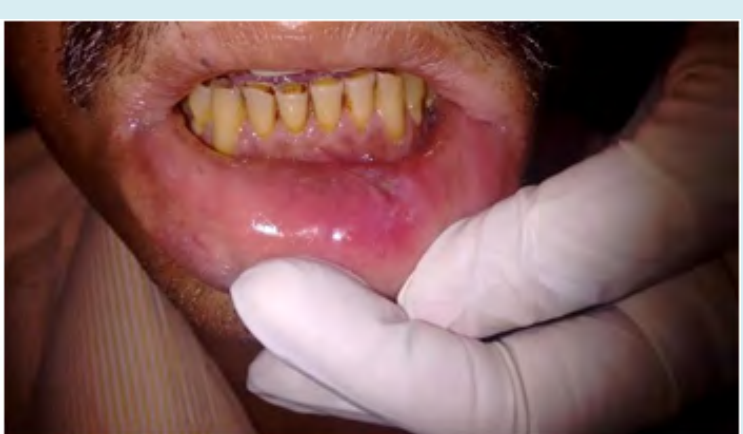

Figure 4: Extraoral photograph showing reduction of lower lip swelling post treatment.

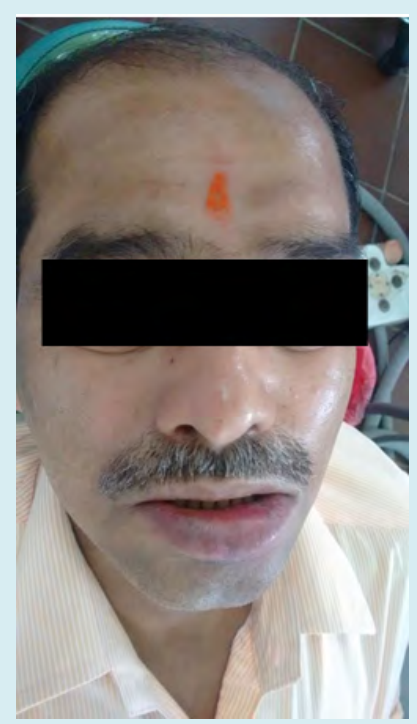

Figure 5: Intraoral image showing healing of lower lip ulcer post treatment.

\section{Discussion}

Insect bite dermatitis has no age or gender predilection, usually occur when patients are asleep at night time. Lymph nodes are not involved in insect bite dermatitis. Insects bites occur on exposed skin surfaces, and the lesions are unilateral in most of the cases [1].

Insect bite lesions are due to reaction of the host or allergy to injected secretions, including venoms (from stinging insects) and enzymes. Histamine, acetylcholine and other substances which act on blood vessels have also been isolated from the venom of stinging insects, and they also may play a role in the immediate allergic reaction. The intensity of the reaction may vary from none to severe depending on the degree of host allergy [2]. 
Common reactions and dermatological findings are urticaria, papules, erythematous and edematous eruptions. In some cases, the toxic venom can lead to angioedema or circulatory collapse and cause significant systemic reactions like autonomic instability, neurotoxicity, organ failure and anaphylactic reactions which can be fatal. In such cases, immediate diagnosis and treatment with epinephrine are crucial. The reaction is due to an allergic response to proteins in the insect's saliva, leading in about three-quarters of all persons to an immediate allergic reaction and in about onehalf to a delayed reaction [3]. Mechanical injury to tissue during bites and stings results in pain and swelling and provides a portal of entry for bacteria which can result in secondary infection In patients presenting with a necrotic ulcer following suspected brown recluse envenomation, clinicians should also consider diagnoses such as pyoderma gangrenosum, staphylococcus or streptococcus skin infection, diabetic ulcers, necrotizing fasciitis, fungal infections, leishmaniasis, and sporotrichosis [4].

\section{Conclusion}

In current case, underlying systemic condition of diabetes mellitus resulted in long standing ulcer and delayed healing.

\section{References}

1. Kar S, Dongre A, Krishnan A, Godse S, Singh N (2013) Epidemiological study of insect bite reactions from Central India. Indian J Dermatol 58(5): 337-341.

2. (2019) Lookingbill and Marks. Principles of Dermatology. $6^{\text {th }}$ (Edn.), pp: 151-165.

3. Przybilla B, Ruëff F (2012) Insect Stings-Clinical Features and Management. Dtsch Arztebl Int 109(13): 238-248.

4. Juckett G (2013) Arthropod bites. Am Fam Physician 88(12): 841-847. 